\title{
Las aves de distribución mediterránea en el País Vasco: abundancia y tendencia poblacional en el sur de Álava.
}

\section{Birds with a Mediterranean distribution in the Basque Country: abundance and population trends in the south of Álava.}

José Antonio Gainzarain ${ }^{1 *}$, Gorka Belamendia²

$z^{*}$

\section{Resumen}

Varias especies de aves, entre ellas algunas catalogadas como amenazadas, presentan en la Comunidad Autónoma Vasca una distribución meridional centrada en la Rioja Alavesa. Mediante transectos efectuados en esta comarca en las primaveras de 2012 y 2013, se obtuvieron datos sobre la abundancia de estas especies en diferentes tipos de hábitat. Con el fin de conocer la evolución reciente de sus poblaciones, esta información se comparó con la de dos estudios anteriores, llevados a cabo en 1988/89 y 1994. Nuestros datos revelan que el alcaudón real Lanius meridionalis se ha extinguido como nidificante en la comarca y que, junto con esta especie, la tórtola europea Streptopelia turtur, la calandria común Melanocorypha calandra y el escribano hortelano Emberiza hortulana son las que han experimentado una disminución más marcada. En sentido contrario, la curruca cabecinegra Sy/via melanocephala ha colonizado la comarca después de 1988, y la terrera común Calandrella brachydactyla probablemente haya aumentado sus efectivos. Las tendencias observadas coinciden en gran medida con las registradas en el conjunto de España mediante el programa SACRE. La abundancia global del grupo de especies analizadas ha sufrido un descenso significativo en las dos últimas décadas, paralelo a un notable aumento de la superficie ocupada por el viñedo en la comarca en detrimento de otros usos minoritarios del suelo.

Palabras clave: Abundancia, evolución poblacional, aves mediterráneas, paisaje agrícola, viñedos, transectos lineales, Rioja Alavesa, País Vasco.

\footnotetext{
1 Instituto Alavés de la Naturaleza

Apdo. de correos $2092 \cdot 01080$ Vitoria-Gasteiz

2 Centro de Estudios Ambientales. Ayuntamiento de Vitoria-Gasteiz

Casa Dehesa de Olarizu, s/n • 01006 Vitoria-Gasteiz

* Correspondencia: j.gainzarain@gmail.com
} 


\begin{abstract}
In the Basque Autonomous Community, several bird species, some of which are considered as threatened, present a southern distribution centered on Rioja Alavesa. By means of line transects in this area, during the springs of 2012 and 2013 data were gathered about the abundance of these species in different habitats. In order to evaluate the recent evolution of their populations, this information was compared to that offered by two former studies, carried out in the same area in 1988/89 and 1994. Our data showed that the Southern grey shrike Lanius meridionalis has disappeared as a breeding bird, and that the European turtle dove Streptopelia turtur, the calandra lark Melanocorypha calandra and the ortolan bunting Emberiza hortulana have experienced a substantial decrease. On the other hand, the Sardinian warbler Sylvia melanocephala was added to the local avifauna after 1988, and the short-toed lark Calandrella brachydactyla population probably has increased. Observed trends match to a great extent with the evolution registered in the whole of Spain by the SACRE monitoring program. Besides specific trends, the global abundance of the analyzed group of southern species has decreased significantly in the last two decades, a process parallel to the increase in the surface occupied by vineyards at the expense of other land uses.
\end{abstract}

Key words: Abundance, population trends, Mediterranean birds, agricultural landscape, vineyards, linear transects, Rioja Alavesa, Basque Country, España.

\title{
Laburpena
}

Hegazti espezie batzuk, horien artean mehatxatu egoeran sailkatutako zenbait, Euskal Autonomia Erkidegoko hegoaldean kokatuta daude, erdigunea Arabako Errioxa dutelarik. 2012 eta 2013ko udaberrietan eskualde horretan egindako trantsektuen bidez espezie horien ugaritasunari buruzko datuak lortu ziren, habitat mota ezberdinetan. Bada, populazio horien azkenaldiko eboluzioa ezagutzearren, informazio hori aurretik 1988/89an eta 1994an egindako beste bi azterlanetakoekin konparatu zen. Gure datuen arabera, antzandobi handia Lanius meridionalis desagertu egin da habiagile gisa; horrekin batera, beste espezie hauek ere nabarmen gutxitu dira: usapal arrunta Streptopelia turtur, kalandria Melanocorypha calandra eta berdantza miarritza Emberiza hortulana. Beste batzuen kasuan, ordea, baliteke kopurua handitu izana, esaterako, txinbo burubeltza Sylvia melanocephala, zeinak 1988az geroztik kolonizatu baitu eskualdea, eta txoriandre arrunta Calandrella brachydactyla. Antzemandako joerak neurri handian bat datoz SACRE programaren bidez Espainia osorako erregistratutakoekin. Oro har, aztertutako espezie taldearen ugaritasuna nabarmen murriztu da azken bi hamarkadetan, mahastiek hartutako azalera handitu ahala, lurraren beste erabilera minoritario batzuk gutxitzearekin batera.

Gako hitzak: ugaritasuna, populazioen eboluzioa, hegazti mediterranearrak, nekazaritza paisaia, mahastiak, trantsektu linealak, Arabako Errioxa, Euskadi.

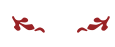




\section{Introducción}

La Comunidad Autónoma del País Vasco (CAPV), situada entre la costa cantábrica y el río Ebro, cuenta con una gran variedad de condiciones climáticas y alberga, en un reducido ámbito espacial, comunidades biológicas con características muy contrastadas (Aseginolaza et al., 1988). En el extremo meridional de la región, al sur de la barrera biogeográfica de la sierra de Cantabria, y en plena depresión del Ebro, la Rioja Alavesa presenta un carácter nítidamente mediterráneo. La mayor parte de esta comarca cuenta con un ombroclima seco y pertenece al piso biogeográfico mesomediterráneo (Loidi et al., 1994), unas condiciones que le separan claramente del resto del territorio y le llevan a acoger a numerosas especies exclusivas en el ámbito de la CAPV (Aseginolaza et al., 1984; Álvarez et al., 1985).

En el caso de las aves, en esta comarca nidifican diversas especies ausentes o muy raras en el resto de la comunidad autónoma. Varias de ellas presentan un estado de conservación desfavorable (Fernández de Mendiola \& Bea, 1998), lo que les llevó a ser incluidas en el Catálogo Vasco de Especies Amenazadas de la Fauna y Flora Silvestre y Marina (Orden de la Consejera de Medio Ambiente y Política Territorial de 18 de junio de 2013). La información sobre la distribución y abundancia de estas especies procede por una parte de los atlas de aves reproductoras (Álvarez et al., 1985; Martí \& del Moral, 2003) y, por otra, de los estudios de Nuevo (1990) y Gainzarain (1996), que aportan información sobre la composición, estructura y selección de hábitat de la comunidad de aves del mosaico agrícola dominante en el paisaje de la Rioja Alavesa.

Desde estos últimos estudios, realizados hace dos décadas, existe una falta casi total de información sobre la situación de las poblaciones reproductoras de estas aves de distribución restringida en la CAPV (la utilidad al respecto del último atlas se ve limitada por el hecho de que casi todas las cuadrículas de la comarca incluyan importantes superficies de provincias limítrofes). Con unos efectivos en general exiguos y especialmente vulnerables, al estar localizados en el límite de su área de distribución (Swihart et al., 2006), es muy posible que la situación poblacional de estas aves haya cambiado sustancialmente en los últimos años. La mayoría de ellas son propias de medios agrícolas, un tipo de hábitat cuya avifauna ha experimentado una tendencia especialmente negativa tanto en el conjunto de Europa (Gregory et al., 2005), como en España (Escandell, 2013) y el País Vasco (Fernández \& Gainzarain, 2004). Varias son además migrantes transaharianas, otro grupo con tendencia negativa en Europa (Sanderson et al., 2006), lo que sugiere asimismo que sus poblaciones en la CAPV pueden haber disminuido en tiempos recientes.

Habida cuenta de esta falta de información, los principales objetivos del presente trabajo son:

1.- Obtener datos actualizados sobre la distribución y abundancia en la Rioja Alavesa de las aves que en la CAPV presentan sus principales poblaciones en esta comarca.

2.- Estimar la tendencia reciente de sus efectivos reproductores mediante la comparación con los estudios previos disponibles. 


\section{Material y métodos}

La Rioja Alavesa ocupa una superficie de $316 \mathrm{~km}^{2}$ y en ella se pueden distinguir dos unidades geográficas claramente diferenciadas: al norte las laderas meridionales de la sierra de Toloño (altitud máxima de 1.454 m s.n.m.), cuyas crestas marcan el límite comarcal, y más al sur el área que corresponde al valle del Ebro, que va desde el piedemonte serrano hasta el curso de este río, en un intervalo de altitudes aproximado de 400-700 m s.n.m. (Fig. 1). Las laderas de la sierra se cubren de un mosaico de bosques juveniles (principalmente de Quercus ilex) y matorral esclerófilo, mientras que en el paisaje de la zona basal predominan las fincas dedicadas al cultivo de la vid, alternando con herbazales ruderales, parcelas de cereal, y rodales de almendros y olivos. En las laderas de los numerosos cerros y barrancos de esta zona aparece un mosaico de pastos xerófilos de Brachypodium retusum y matorrales de bajo porte dominados por Genista scorpius, Thymus vulgaris, Rosmarinus officinalis y Quercus coccifera. En el fondo de los barrancos, las márgenes de los arroyos, de corto recorrido y reducido caudal, se cubren de matorrales de zarzas (Rubus spp.) y sotos discontinuos de arbolado caducifolio bastante degradado.

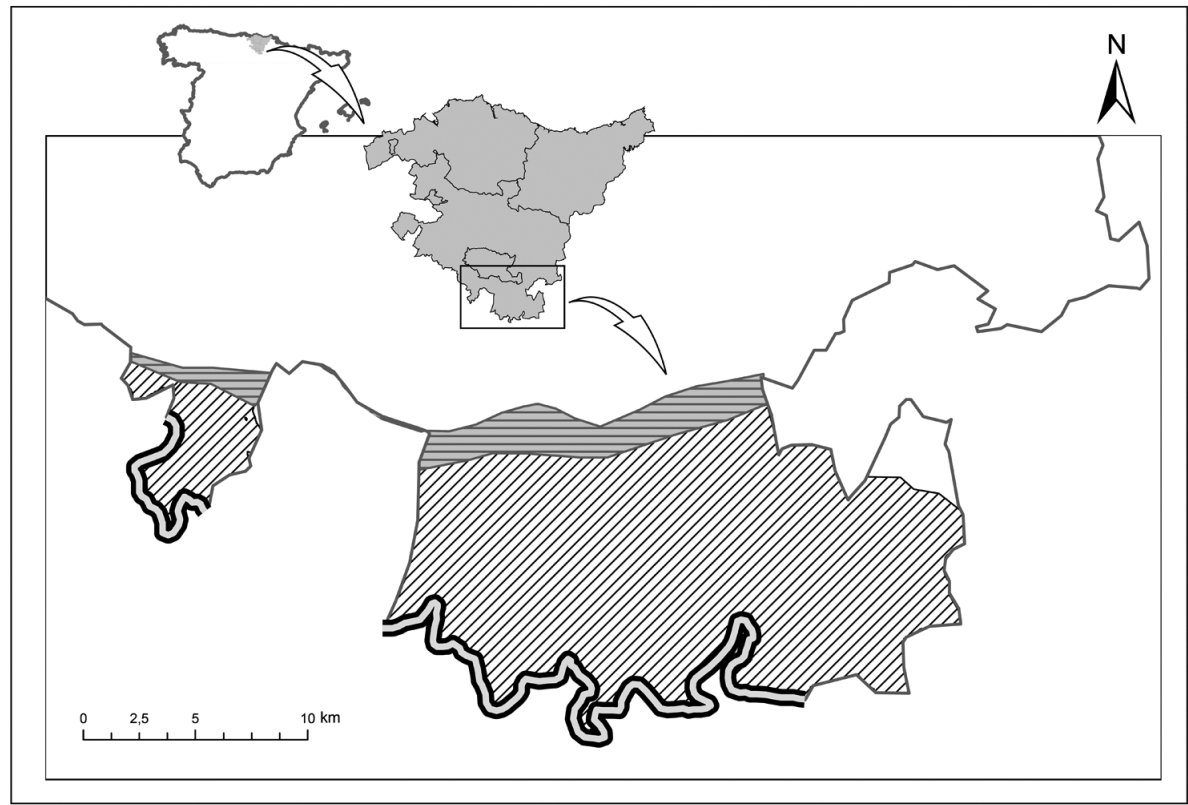

Fig. 1.- Localización del área de estudio. En trama horizontal se destacan las laderas de la sierra de Toloño, en trama oblicua el mosaico de viñedos de la zona basal de la Rioja Alavesa, y en línea doble el río Ebro.

Fig. 1.- Geographical location of the study area. Toloño range slopes are shaded horizontally, and obliquely the lowland vineyard mosaic of the Rioja Alavesa. A double line marks the Ebro river course. 
En el límite sur de la comarca, el río Ebro presenta una vegetación ribereña mejor desarrollada, por lo general una estrecha banda de bosque galería que en algunos enclaves alcanza un buen desarrollo y que coexiste con plantaciones de chopos Populus x canadensis de reducida extensión.

Aunque en los muestreos realizados se registraron todas las especies de aves presentes, aquellas de interés para el presente trabajo fueron las que en el País Vasco cuentan con una distribución meridional y mantienen sus principales poblaciones en la Rioja Alavesa (aunque en algunos casos puedan ser localmente comunes en el resto del territorio, como es el caso de las currucas cabecinegra y rabilarga). Se trata de veinte especies (catorce de las cuales están incluidas en el Catálogo Vasco de Especies Amenazadas), que se relacionan a continuación siguiendo la nomenclatura de Gutiérrez et al. (2012):

- Especies vulnerables: abubilla (Upupa epops), alcaudón real (Lanius meridionalis), y alcaudón común (Lanius senator).

- Especies de interés especial: abejaruco europeo (Merops apiaster), terrera común (Calandrella brachydactyla), calandria común (Melanocorypha calandra), cogujada montesina (Galerida theklae), bisbita campestre (Anthus campestris), collalba rubia (Oenanthe hispanica), curruca carrasqueña (Sylvia cantillans), curruca tomillera (Sylvia conspicillata), curruca mirlona (Sylvia hortensis), pájaro-moscón europeo (Remiz pendulinus), y escribano hortelano (Emberiza hortulana).

- Otras: paloma zurita (Columba oenas), tórtola europea (Streptopelia turtur), cogujada común (Galerida cristata), zarcero bereber (Iduna opaca), curruca rabilarga (Sylvia undata), y curruca cabecinegra (Sylvia melanocephala).

Para censar la comunidad de aves de la Rioja Alavesa se efectuaron un total de 113,5 km de transectos divididos en unidades de 500 m. Estos se cubrieron en mayo y la primera quincena de junio de 2012 y 2013, entre treinta minutos y cuatro horas tras la salida del sol, y en condiciones meteorológicas favorables: ausencia de lluvia o viento fuerte. En ellos se registraron todas las aves vistas u oídas, separando los contactos obtenidos dentro y fuera de una banda de 25 m a cada lado de la línea de progresión (transecto finlandés; Tellería, 1986). Cuando las condiciones lo permitieron se procuró llevar a cabo los transectos campo a través, evitando seguir caminos y pistas, con el fin de que el terreno cubierto fuera lo más representativo posible del conjunto del paisaje de la zona. Los recorridos se repartieron del siguiente modo:

- 146 transectos (73 km) en el mosaico agrícola de la zona.

- 55 transectos $(27,5 \mathrm{~km}$ ) en los matorrales y carrascales de la solana de la sierra de Toloño.

- 26 transectos $(13 \mathrm{~km}$ ) en las riberas del río Ebro a su paso por la Rioja Alavesa (en este medio de naturaleza lineal, que frecuentemente no alcanza los 25 m de anchura, solo se registraron las aves que hacían uso de la vegetación de ribera). 
Con el fin de distribuir espacialmente los muestreos de modo uniforme, se tomaron como referencia las siete cuadrículas UTM de $100 \mathrm{~km}^{2}$ que abarca el área de estudio, en las que se calculó la cobertura de hábitats utilizando la cartografía EUNIS (Gobierno Vasco, 2007). Para cada tipo de hábitat, el reparto de itinerarios por cuadrícula fue proporcional al porcentaje que del total comarcal correspondía a cada una de ellas.

Fuera de estos muestreos, durante las primaveras del periodo de estudio se efectuaron diversas visitas adicionales a varias localidades de la comarca con el fin de conseguir más datos sobre aquellas especies muy escasas para las que se preveía obtener pocos registros en el curso de los recorridos.

Para estudiar la evolución reciente de las poblaciones de aves mediterráneas en el área de estudio se compararon los resultados obtenidos en el presente trabajo con los correspondientes a dos publicaciones que se remontan aproximadamente a dos décadas atrás:

a) Nuevo (1990) presenta los resultados de $14,5 \mathrm{~km}$ de taxiados llevados a cabo en medios agrícolas de la Rioja Alavesa en las primaveras de 1988 y 1989. Para las especies de interés, se comparó el no total de aves registrado por kilómetro de recorrido (IKA) de este estudio con el obtenido en nuestros transectos. Aunque se trata de un índice de abundancia relativa, el IKA se obtiene a partir del total de contactos, por lo que se adecúa más a estas comparaciones que una estima de abundancia absoluta como es la densidad en la banda principal de muestreo. Su utilización permite por una parte maximizar el tamaño de muestra y, por otra, eliminar el sesgo potencial derivado de la variabilidad entre observadores en el cálculo de distancias (Buchanan et al., 2006).

b) También contamos con los datos inéditos de 1994 en los que se basó el estudio de Gainzarain (1996) sobre la selección de hábitat de la avifauna del paisaje agrícola de la comarca. En este caso, el trabajo de campo consistió básicamente en la descripción del hábitat en un radio de 25 m en torno a cada ave o bando detectados a lo largo de diversos recorridos repartidos por el área de estudio. El número total de ejemplares registrado de este modo fue de 1.240, de los que 296 (23,87\%) correspondieron a las especies objeto de interés en el presente trabajo.

Estos dos estudios cubrieron únicamente el mosaico agrícola de la comarca, por lo que para las comparaciones con nuestros datos solo se han utilizado los referidos a este hábitat: 146 unidades de 500 m en 2012/13, en las que se detectaron un total de 2.901 ejemplares, 537 de ellos (18,51\%) de las especies de interés.

En las comparaciones entre estudios se empleó el método del bootstrapping (Greenwood, 1991), frecuentemente usado para el cálculo de intervalos de confianza en estimas poblacionales de aves (p. ej. Wotton et al., 2000). Para la comparación con los resultados de Nuevo (1990), del total de unidades de 500 m cubiertas en 2012/13 se extrajo al azar con repetición una muestra de 29, equivalente a los 14,5 km del citado estudio, y se calculó el IKA correspondiente. Tras repetir este proceso 10.000 veces se obtuvo una distribución de 
abundancias para cada especie, de la que posteriormente se calcularon los cuantiles $2,5 \%(250)$ y $97,5 \%(9.750)$ con el fin de establecer los límites de confianza al 95\%. Para cada especie, la diferencia entre ambos estudios se consideró como significativa si el valor de abundancia obtenido en 1988/89 se encontraba fuera de estos valores límite. En la comparación con los datos de Gainzarain (1996), y dado que en este estudio no se registró la distancia recorrida, se tomó como referencia el número total de aves detectadas (1.240). En nuestros recorridos, con una media de 19,87 ejemplares por unidad de 500 m, esto corresponde a un total de 62 unidades, que son las que se extrajeron mediante el procedimiento ya descrito para obtener una nueva distribución de abundancias. El bootstrapping se efectuó en esta ocasión a partir del número de individuos de cada especie, y los intervalos de confianza obtenidos se refirieron a la media de esta variable. Para convertir estos valores en porcentaje sobre el total se dividieron entre el número medio total de aves por muestreo $(19,87)$ y se multiplicó el resultado por cien. El método descrito para las comparaciones con uno y otro estudio se repitió finalmente con la abundancia total del conjunto de especies tratadas.

Para cada especie se calculó además su correspondiente tasa de variación anual, expresada en porcentaje a partir de la ecuación de la tasa de crecimiento geométrico:

Tasa de variación anual $=\left(\left[a b_{1} / a_{2}\right]^{1 / n}-1\right) \times 100$

Siendo ab 1 la abundancia hallada en el presente estudio (IKA en la primera comparación, y porcentaje sobre el total de individuos en la segunda), $\mathrm{ab}_{2}$ la abundancia del estudio precedente, y n el intervalo de años transcurrido entre ambos: 23,5 desde Nuevo (1990) y 18 desde Gainzarain (1996) (la gran mayoría de los recorridos del mosaico agrícola se llevaron a cabo en 2012 y ese fue el año que se tomó como base para este cálculo). La tasa final de variación anual para cada especie resulta del promedio de las comparaciones con ambos estudios previos. Dado que el cálculo de esta tasa no se adecúa a los valores de abundancia cero, en los casos en que una especie no se registró en alguno de los estudios considerados, su abundancia se consideró como la equivalente a la detección de un solo individuo en el conjunto de los muestreos correspondientes. En el caso de Nuevo (1990), esto supone un valor de IKA de 0,06 ind/km, y en el presente estudio un IKA de 0,014 ind/km y un 0,034\% del total de contactos. Los datos de Gainzarain (1996) no precisaron en ningún caso de esta modificación.

Mediante un test de la $U$ de Mann-Whitney se compararon los valores de estas tasas de variación anual entre diversos grupos de especies definidos según su dieta (insectívoros vs granívoros), sus hábitos migratorios (migrantes transaharianos vs sedentarios y migrantes presaharianos) y sus preferencias de hábitat en la comarca (medios agrícolas vs matorral esclerófilo; según Gainzarain (1996) y datos propios inéditos) (ver Tabla 2). Asimismo, se calculó con un test de Spearman la correlación entre esta tasa de variación anual y el índice de evolución media interanual de cada especie entre 1998 y 2012 para el conjunto de España (según los datos del programa SACRE; Escandell, 2013). Todos los test estadísticos se efectuaron mediante el programa R 3.0.2. (R Core Team, 2013). 


\section{Resultados}

En los itinerarios de censo se detectaron diecisiete de las veinte especies consideradas en el presente trabajo (Tabla 1). La zona basal de la Rioja Alavesa acoge a todas ellas: dieciséis fueron registradas en el mosaico agrícola de la zona (todas menos el pájaro moscón, que solo apareció - muy escaso- en el Ebro), y seis en los sotos del Ebro. A las laderas de la sierra de Toloño llegan nueve especies, si bien dos de ellas -el abejaruco europeo y el escribano hortelano- únicamente se encontraron a baja altitud en el extremo occidental de esta alineación, en el entorno del desfiladero de las Conchas de Haro (WN11).

Las visitas adicionales posibilitaron la detección de algunos ejemplares de zarcero bereber: sendos machos en arroyos con buena cobertura de matorral en las localidades de Viñaspre, Barriobusto y Yécora, en el sector oriental de la comarca (WN41). También sirvieron para obtener más registros de calandria común y escribano hortelano. La primera especie solamente apareció en uno de nuestros transectos, en el que se observaron cuatro machos cantando en un rastrojo de cereal no lejos de Oyón (WN40). Fuera de los transectos, se localizaron tres machos en Oyón en 2012, y sendos machos en las inmediaciones de El Campillar (WN30) y de Lanciego (WN41) en 2013. El escribano hortelano por su parte apareció en los muestreos únicamente en cuatro ocasiones, en laderas de pastos y matorral mediterráneo del entorno del Ebro (WN20 y 30) y de las Conchas de Haro (WN11). En las visitas adicionales se ha localizado en tres puntos más de las cercanías de Oyón (WN40). No se obtuvo ningún registro de otras dos especies ausentes en los itinerarios: la curruca tomillera y el alcaudón real (la distribución por cuadrículas de todas las especies puede consultarse en el Apéndice 1).

Diez especies alcanzaron sus mayores abundancias en el mosaico agrícola; del resto, la paloma zurita, la tórtola europea y el pájaro moscón lo hicieron en las riberas del Ebro, y el escribano hortelano y las currucas rabilarga, carrasqueña y mirlona en las laderas de la sierra de Toloño. Con la excepción de la cogujada común en el mosaico agrícola, y de la curruca cabecinegra, tanto en este medio como en la sierra, todas estas aves de distribución mediterránea resultaron ser escasas en la comarca, con IKAs inferiores a 1 ind $/ \mathrm{km}$.

Las variaciones en la abundancia relativa de las diferentes especies con respecto a estudios anteriores figuran en la Tabla 2. Destaca por una parte la curruca cabecinegra, que no se detectó en la zona en 1988/89 y resultó ser la especie más abundante en 2012/13, y por otra el alcaudón real, registrado en los dos estudios anteriores, pero que no aparece en nuestros muestreos. En relación con los taxiados de 1988/89, seis especies disminuyen significativamente su abundancia y dos la aumentan, cifras que se repiten en la comparación con los datos de 1994. Las especies con cambios más evidentes son la tórtola europea, la calandria común, el alcaudón real y el escribano hortelano (que disminuyen en los dos casos) y la curruca cabecinegra (que aumenta en ambos). Con valores elevados de la tasa media de variación anual, aunque con diferencias significativas únicamente en la comparación con los datos de 1994 (los de mayor tamaño de muestra), destacan la 


\begin{tabular}{|lccccc|}
\hline \multirow{2}{*}{ Especie } & \multicolumn{2}{c}{ M. AGR. } & \multicolumn{2}{c}{ SIERRA } & RIBERA \\
& DENS. & IKA & DENS. & IKA & IKA \\
\hline Columba oenas & - & 0,08 & - & - & 0,31 \\
Streptopelia turtur & 0,11 & 0,25 & - & 0,29 & 0,54 \\
Merops apiaster & 0,33 & 0,96 & - & 0,33 & - \\
Upupa epops & 0,11 & 0,29 & - & 0,11 & - \\
Melanocorypha calandra & - & 0,05 & - & - & - \\
Calandrella brachydactyla & 0,38 & 0,59 & - & - & 0,08 \\
Galerida theklae & 0,35 & 0,40 & - & - & - \\
Galerida cristata & 0,38 & 1,15 & - & - & - \\
Anthus campestris & 0,44 & 0,70 & 0,13 & 0,15 & - \\
Oenanthe hispanica & 0,35 & 0,55 & - & - & - \\
Sylvia undata & 0,31 & 0,25 & 0,07 & 0,36 & - \\
Sylvia cantillans & 0,20 & 0,14 & 1,01 & 0,95 & - \\
Sylvia melanocephala & 1,59 & 1,75 & 0,72 & 1,02 & 0,08 \\
Sylvia hortensis & 0,08 & 0,05 & 0,07 & 0,40 & - \\
Remiz pendulinus & - & - & - & - & 0,15 \\
Lanius senator & 0,06 & 0,11 & - & - & 0,08 \\
Emberiza hortulana & - & 0,03 & 0,07 & 0,07 & - \\
\hline
\end{tabular}

Tabla 1.- Valores de densidad (ind/10 ha) y del Índice Kilométrico de Abundancia (ind/km) de las especies de aves consideradas en el presente estudio. M. AGR.: mosaico agrícola del valle del Ebro; SIERRA: ladera sur de la sierra de Toloño; RIBERA: orillas alavesas del río Ebro.

Table 1.- Density (ind/10 ha) and Kilometric Abundance Index (ind/km) of the bird species included in this study. M. AG. Agricultural mosaic of the Ebro Valley; SIERRA: southern slopes of Toloño range; RIBERA: Ebro River banks in Álava.

terrera común, con tasa de variación positiva, y, con valores negativos, la curruca rabilarga. Más allá de las variaciones específicas encontradas, nuestros resultados muestran una disminución de la abundancia global del grupo de especies consideradas, con diferencias significativas en los dos test realizados: el IKA pasa de 11,71 a 7,37 ind/ km, y la proporción sobre el total del 23,87 al 18,51\% (Tabla 2).

La tasa media de variación anual de cada especie se correlaciona significativamente con la del programa SACRE para el conjunto de España $\left(r_{S}=0,533 ; P=0,028 ; n=17 ;\right.$ Fig. 2). En lo que respecta a los distintos grupos de especies, esta tasa es negativa en todos los casos, pero no se han encontrado diferencias significativas entre ellos en relación con 


\begin{tabular}{|lcccccccc|}
\hline Especie & CAT & IKA $_{88 / 89}$ & $\begin{array}{c}\text { IKA }_{12 / 13} \\
(\text { IC95\%) }\end{array}$ & TVA1 & $\% 94$ & $\begin{array}{c}\% 12 / 13 \\
\text { (IC95\%) }\end{array}$ & TVA2 & TVMA \\
\hline C. oenas & GSA & 0,41 & $\underline{0-0,28}$ & $-6,7 \%$ & 0,24 & $0-0,57$ & $-0,7 \%$ & $-3,7 \%$ \\
S. turtur & GTA & 0,89 & $\underline{0-0,55}$ & $-5,3 \%$ & 2,66 & $\underline{0,24-1,14}$ & $-7,8 \%$ & $-6,5 \%$ \\
M. apiaster & ITA & 0,75 & $0,35-1,79$ & $1,1 \%$ & 1,05 & $1,30-3,82$ & $4,7 \%$ & $2,9 \%$ \\
U. epops & ITA & 0,48 & $0-0,62$ & $-2,1 \%$ & 0,56 & $0,24-1,38$ & $1,5 \%$ & $-0,3 \%$ \\
M. calandra & GSA & 0,75 & $\underline{0-0,28}$ & $-10,9 \%$ & 2,82 & $\underline{0-0,65}$ & $-15,4 \%$ & $-13,1 \%$ \\
C. brachydactyla & GTA & 0,34 & $0,21-1,17$ & $2,4 \%$ & 0,40 & $\underline{0,73-2,35}$ & $7,9 \%$ & $5,1 \%$ \\
G. theklae & GSM & 0 & $\underline{0,07-0,47}$ & $8,4 \%$ & 1,13 & $0,32-2,03$ & $-0,7 \%$ & $3,9 \%$ \\
G. cristata & GSA & 5,79 & $\underline{0,55-1,86}$ & $-6,6 \%$ & 2,82 & $1,79-4,14$ & $0,2 \%$ & $-3,2 \%$ \\
A. campestris & ITA & 0,55 & $0,28-1,24$ & $1,0 \%$ & 1,37 & $0,97-2,60$ & $1,4 \%$ & $1,2 \%$ \\
O. hispanica & ITA & 0,62 & $0,14-1,10$ & $-0,5 \%$ & 0,81 & $0,65-2,27$ & $3,0 \%$ & $1,2 \%$ \\
S. undata & ISM & 0,34 & $0-0,55$ & $-1,3 \%$ & 5,73 & $\underline{0,16-1,14}$ & $-11,6 \%$ & $-6,5 \%$ \\
S. cantillans & ITM & 0,20 & $0-0,35$ & $-1,5 \%$ & 1,29 & $\underline{0,08-0,65}$ & $-7,0 \%$ & $-4,2 \%$ \\
S. melanocephala & ISM & 0 & $\underline{1,03-2,55}$ & $15,4 \%$ & 1,53 & $\underline{3,17-5,68}$ & $6,1 \%$ & $10,8 \%$ \\
S. hortensis & ITM & 0,06 & $\underline{0-0,21}$ & $-0,8 \%$ & 0,16 & $\underline{0-0,41}$ & $-0,7 \%$ & $-0,8 \%$ \\
L. meridionalis & ISM & 0,13 & $\underline{0-0}$ & $-3,2 \%$ & 0,48 & $\underline{0-0}$ & $-13,5 \%$ & $-8,4 \%$ \\
L. senator & ITA & 0,06 & $0-0,35$ & $2,6 \%$ & 0,40 & $0-0,65$ & $-2,0 \%$ & $0,3 \%$ \\
E. hortulana & GTM & 0,34 & $\underline{0-0,14}$ & $-9,8 \%$ & 0,48 & $\underline{0-0,46}$ & $-9,6 \%$ & $-9,7 \%$ \\
\hline TOTAL & & 11,71 & $\underline{5,52-9,31}$ & & 23,87 & $\underline{15,26-21,92}$ & & \\
\hline
\end{tabular}

Tabla 2.- Variación en la abundancia relativa de las diferentes especies. IKA88/89: ind/km según Nuevo (1990); IKA 12/13 (IC95\%): intervalos de confianza al 95\% del IKA obtenido en el presente estudio; \%94: porcentaje del total de individuos registrados en Gainzarain (1996); \%12/13 (IC95\%): intervalos de confianza al $95 \%$ del porcentaje del total de individuos registrados en el presente estudio; TVA1 y TVA2: tasa de variación anual en una y otra comparación (véase Métodos). TVMA: tasa de variación media anual (promedio de TVA1 y TVA2). Aparecen subrayados los intervalos de confianza con diferencias significativas respecto al correspondiente estudio previo. En la segunda columna (CAT) se señalan las categorías en que se ha incluido a cada especie según su dieta (l: insectívoros, G: granívoros), sus hábitos migratorios (T: migrantes transaharianos, S: sedentarios/ presaharianos), y sus preferencias de hábitat en la comarca (A: medios agrícolas, M: matorral esclerófilo).

Table 2.- Variation in the relative abundance values of the different bird species. IKA88/89: ind/km from Nuevo (1990); IKA12/13 (IC95\%): 95\% confidence intervals of the Kilometric Abundance Index obtained in this study; \%94: percentage of the total number of individuals recorded by Gainzarain (1996); \%12/13 (IC95\%): 95\% confidence intervals of the percentage of the total number of individuals recorded in this study; TVA1 and TVA2: annual change rate in each comparison (see Methods). TVMA: average annual change rate (mean of TVA1 and TVA2). The underlining highlights the confidence intervals with significant differences in relation to the corresponding previous study. The second column (CAT) features the categories in which each species has been included according to its diet (I: insectivorous, G: granivorous), migratory status (T: transaharian migrants, S: sedentary/ presaharian migrants), and habitat preferences in the area (A: agricultural habitats, M: sclerophyllous scrub). 


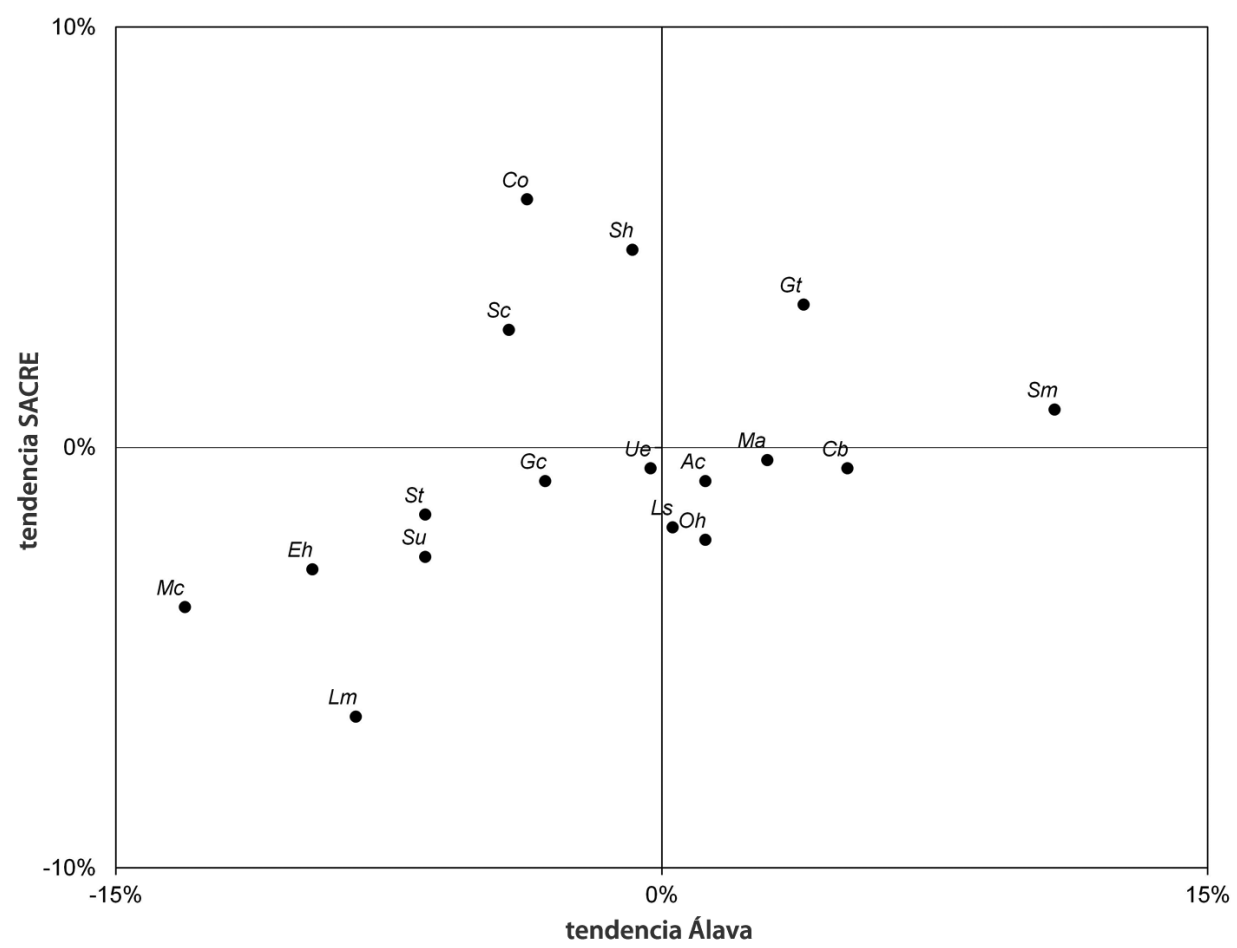

Fig. 2.- Relación entre las tasas de variación anual de las diferentes especies obtenidas para la Rioja Alavesa en el presente trabajo (tendencia Álava), y en el conjunto de España en el programa SACRE (tendencia SACRE). La especie a la que corresponde cada punto se identifica mediante las iniciales de su nombre científico.

Fig. 2.- Relationship between the annual change rates of the different species obtained in Rioja Alavesa by this study (tendencia Álava), and in the whole of Spain by the SACRE project (tendencia SACRE). Species are identified by the initials of their scientific names.

la dieta (granívoros: -3,88\%; insectívoros: -0,37\%; test de la $U$ de Mann-Whitney: $U=25,5$, $P=0,328$ ), los hábitos migratorios (transaharianos: $-1,08 \%$; sedentarios/ presaharianos: $-2,89 \% ; U=27,5, P=0,494$ ), o las preferencias de hábitat (medios agrícolas: -1,61\%; medios naturales; $-2,13 \% ; U=28,5, P=0,558)$. 


\section{Discusión}

\section{Aspectos metodológicos}

La evolución de la abundancia de las especies tratadas en este estudio se ha abordado mediante la comparación con los estudios previos de Nuevo (1990) y Gainzarain (1996). En este último caso, en el que se usó una metodología muy diferente de la del presente trabajo, es posible que los resultados se hayan visto afectados por las variaciones en detectabilidad de las diversas especies, ya que en el estudio de 1996 solamente se incluyeron los registros de ejemplares cuya posición perfectamente localizada posibilitara la descripción del hábitat a su alrededor. La metodología del transecto finlandés que hemos utilizado en nuestros muestreos no cuenta con esta limitación, de manera que las especies detectables a mayor distancia podrían estar sobrerrepresentadas con relación al anterior trabajo, con lo que su evolución en el intervalo entre ambos tendería a mostrar un falso signo positivo. No obstante, los datos no reflejan este fenómeno, ya que la detectabilidad de cada especie -cuantificada como el inverso del porcentaje sobre el total del número individuos registrado dentro de la banda de muestreo de $50 \mathrm{~m}$ (para todas las especies con al menos diez ejemplares censados en el total de los itinerarios de 1988/89 y 2012/13) - no muestra correlación con el cambio registrado en su abundancia entre 1994 y $2012\left(r_{s}=0,013 ; P=0,964 ; n=14\right)$.

Otro potencial sesgo metodológico se deriva de que el índice utilizado en la comparación con Gainzarain (1996) -porcentaje de individuos de una especie sobre el total de ejemplares- depende tanto de la abundancia de la especie analizada como de la del resto. Si la población de esa especie ha permanecido constante en el periodo considerado pero la abundancia de la comunidad de aves en su conjunto ha variado, la comparación de los índices respectivos mostraría un cambio que no se habría producido en realidad. De hecho, se puede dar por seguro que la densidad total no ha permanecido constante en el intervalo de dieciocho años considerado, por lo que hay que contar con esta fuente de error a la hora de interpretar los datos. Sin embargo, dado que el valor de densidad total en 2012/13 (25,83 ind/10 ha) es similar al obtenido por Nuevo (1991) 24 años antes $(29,65)$, cabe deducir que este parámetro ha permanecido razonablemente constante en el periodo considerado, y que sus cambios no han afectado de modo sustancial a los índices de variación obtenidos.

Estas y otras diferencias relativas al esfuerzo de muestreo, la metodología y la localización de los recorridos han podido afectar en un grado desconocido a nuestros resultados, aunque la fiabilidad de las tendencias observadas se ve respaldada tanto por la estrecha relación que hemos encontrado entre las tasas anuales de variación resultantes de las comparaciones con uno y otro estudio previo $\left(r_{S}=0,632 ; P<0,01 ; n=17\right)$, como por el hecho de que estas sean consistentes con los resultados obtenidos en el conjunto de España por el programa SACRE. 


\section{Evolución de la avifauna}

La composición del grupo de especies de distribución mediterránea presentes en la Rioja Alavesa resulta similar a la descrita treinta años atrás en el atlas de vertebrados de la CAPV (Álvarez et al., 1985). La incorporación de la curruca cabecinegra, que ha colonizado rápidamente la comarca a partir de la primera observación en 1992 (Gainzarain \& Pérez de Ana, 1995), y la aparente extinción del alcaudón real suponen las variaciones más destacables. Además, es posible que una nueva especie se haya incorporado a la avifauna de la zona: el zarcero bereber, que no fue localizado en el atlas de 1985 ni figura en la revisión sobre vertebrados amenazados del País Vasco de Fernández de Mendiola \& Bea (1998). Se conocía una observación anterior de esta especie en la Rioja Alavesa, en el río Ebro (Gainzarain, 2011), y los nuevos registros de 2012/13 apuntan a que la especie nidifica en la comarca. Es posible que se trate de un colonizador reciente aunque, dada su escasez y su comportamiento retraído, no es descartable que pudiera estar presente con anterioridad. Otra especie que no aparecía en Álvarez et al. (1985) -la curruca tomillera-, cuenta con unas pocas observaciones previas en época de nidificación: un ejemplar en Labastida en 2001 (J. Ma Fernández, com. pers.), y otro en Oyón en 2006 (F. Silván, com. pers.), pero no ha sido detectada en 2012/13 ni tenemos noticia de registros posteriores a 2006.

El alcaudón real es la única especie que parece haber desaparecido en la zona en las últimas décadas. En el atlas de los 80, Álvarez et al. (1985) lo encuentran bien distribuido en la Rioja Alavesa e incluso aportan algunas citas dispersas en la zona media de Álava, pero en el presente trabajo no se ha detectado ningún ejemplar a pesar del elevado esfuerzo de muestreo. Revisando nuestros datos y consultando con otros ornitólogos conocedores de la zona, no hemos encontrado ninguna cita en época de cría posterior a 2006. Así pues, parece lo más probable que en los últimos años el alcaudón real se haya extinguido en la comarca y, por ende, en la Comunidad Autónoma Vasca. Teniendo en cuenta el desplome de su población en el conjunto de España (un 57\% menor en 2012 que en 1998; Escandell, 2013), no sorprende la acusada rarefacción e incluso la desaparición de la especie en zonas del borde de su área de distribución como es el caso del País Vasco. Una tendencia regresiva se ha detectado también en las vecinas provincias de La Rioja (Gámez et al., 2010) y Burgos (Román et al., 1996), así como en el sector más norteño de su área de distribución en Cataluña (Estrada et al., 1999). Estudios genéticos recientes (Olsson et al., 2010) apuntan a que los alcaudones reales ibéricos y del sur de Francia (donde la población de la especie también está disminuyendo; Dubois et al., 2008), podrían constituir una especie diferenciada, lo que añadiría gravedad a su acelerado proceso de regresión.

Las comparaciones con los estudios previos sobre la avifauna de la comarca revelan descensos significativos de la abundancia relativa de otras tres especies: la tórtola europea, la calandria común y el escribano hortelano. La distribución en la CAPV de la más escasa de ellas, la calandria común, se limita en la actualidad al sector suroriental de la Rioja Alavesa, y ya Fernández de Mendiola \& Bea (1998) destacaban en los años 90 su rareza. Su acusada disminución coincide con lo observado para el conjunto de España, donde la población reproductora ha decrecido un 34\% de 1998 a 2012 (Escandell, 2013). 
El escribano hortelano por su parte, también presenta una distribución en la Comunidad Autónoma Vasca restringida a la Rioja Alavesa. Aunque nunca ha sido un ave abundante en la zona (Álvarez et al., 1985), la comparación con estudios previos refleja una disminución muy notable de sus efectivos en las dos últimas décadas, que le ha llevado a estar actualmente al borde de la desaparición en la CAPV. Su población en España ha sufrido un declive moderado (-13,2\% entre 1998 y 2013, Escandell, 2013), pero en la Europa templada sus efectivos han disminuido de forma alarmante y se han producido numerosas extinciones locales (Menz \& Arlettaz, 2012).

Más abundante que las dos especies precedentes, la tórtola europea se repartía como nidificante por gran parte de la superficie de la CAPV hasta fechas no lejanas (Álvarez et al., 1985). Hoy día sin embargo su presencia se limita al sur y al suroeste de Álava, siendo rara fuera de la Rioja Alavesa (Sáenz de Buruaga et al., 2012). Aunque se halla bien distribuida en esta comarca, nuestros datos revelan un descenso muy acusado de sus efectivos, de manera que hoy día resulta una especie escasa, que se concentra principalmente en bosquetes y en arboledas fluviales, tal como señalan Sáenz de Buruaga et al. (2012).

Aunque las diferencias observadas solo llegan a alcanzar la significación estadística en una de las comparaciones realizadas, cabe destacar el caso de la terrera común, según nuestros datos la especie con una evolución más positiva tras la curruca cabecinegra. En el atlas de nidificantes de los 80 (Álvarez et al., 1985) aparece como un ave extremadamente escasa en la Rioja Alavesa, con presencia en sólo dos localidades, mientras que actualmente su población asciende sin duda a varios cientos de parejas, que ocupan sobre todo viñedos y se reparten por casi toda la comarca. Es reseñable sobre todo que esta aparente evolución positiva contrasta con la situación de la especie en el conjunto de España -donde el Libro Rojo de las Aves de España (Madroño et al., 2004) la considera como Vulnerable- o en Cataluña, cuya población se considera como En peligro crítico (Anton et al., 2013). Es posible que el hecho de que las terreras de la Rioja Alavesa ocupen principalmente viñedos explique la mejor situación de su población en relación con otras regiones, en las que la especie se presenta sobre todo en medios de vegetación herbácea como tomillares, pastizales o cultivos cerealistas de secano (Suárez et al., 2002; Estrada et al., 2004).

Tampoco llega a alcanzar la significación estadística la disminución que se observa en la densidad relativa de la curruca rabilarga con respecto a los datos de 1988/89. Sin embargo, la comparación con los datos de 1994 sí revela un declive muy importante de la especie en la Rioja Alavesa; de hecho, Gainzarain (1996) la encuentra como la cuarta especie más abundante de la comarca, mientras que en nuestros recorridos ocupa el lugar veintidós en cuanto a densidad relativa, siendo una especie rara fuera de las zonas más próximas al río Ebro. Habida cuenta del notable descenso poblacional que la especie ha experimentado en el conjunto de España (Escandell, 2013), su declive en el área de estudio parece muy probable.

En el extremo meridional de la Comunidad Autónoma Vasca, las especies de aves mediterráneas presentan tendencias poblacionales dispares, aunque predominantemente negativas, que tienden a ser coincidentes con las del conjunto de España (Escandell, 2013). 
El declive poblacional de este grupo de especies contrasta con lo que sería predecible en un escenario de cambio climático global, que en Europa occidental se prevé ocasione un desplazamiento hacia el norte de las áreas de distribución de la avifauna (Huntley et al., 2007). Dado que en el área de estudio las especies analizadas se encuentran en el límite septentrional de su distribución o están próximas a él, cabría esperar que el reciente proceso de aumento de las temperaturas se tradujera en un incremento de su densidad y una expansión hacia el norte, solo apreciable en nuestros datos en el caso de la curruca cabecinegra y, posiblemente, la terrera común. Factores relacionados con la ecología de cada especie, como la disponibilidad y calidad del hábitat, pueden ser más determinantes en las tendencias observadas que los efectos del cambio climático (Seoane \& Carrascal, 2008). Así, el acelerado proceso de intensificación de los agrosistemas de Europa occidental ha provocado la disminución de las poblaciones de diversas aves especialistas de estos medios (Donald et al., 2001, 2006; Gregory et al., 2005), dando origen a tendencias opuestas a las esperadas según las predicciones basadas en el cambio climático global (Kampichler et al., 2012).

En España, donde el grupo de aves de medios agrícolas es el que presenta una evolución reciente más desfavorable (Escandell, 2013), se cuenta con muy poca información sobre el efecto del proceso de intensificación agrícola sobre sus poblaciones (Santos \& Suárez, 2005), y la mayor parte del esfuerzo investigador se ha centrado en medios cerealistas y esteparios (p. ej. Díaz et al., 1993; Martínez \&Tapia, 2002). A pesar del protagonismo del viñedo en el paisaje de numerosas regiones ibéricas, sus comunidades de aves apenas han sido estudiadas (Suárez, 2004), y los posibles efectos sobre ellas de las nuevas prácticas agrícolas son desconocidos (véase sin embargo Duarte et al., 2014).

En el área de estudio los datos de los últimos censos agrarios (fuente: www.eustat.es) revelan un notable aumento de la extensión dedicada al cultivo de la vid en detrimento de otros usos del suelo, con la consiguiente simplificación del paisaje agrícola. Mientras que la superficie agrícola utilizada (SAU) en la Rioja Alavesa se mantuvo constante entre 1989 y 2009 (de 17.357 a 17.166 ha), la superficie de viñedo aumentó un 27,4\% (de 10.469 a 13.336 ha). Otros aprovechamientos agrícolas minoritarios disminuyeron notablemente en el mismo periodo: el cereal un 37,2\% (de 4.056 a 2.547 ha), los barbechos un 51,5\% (de 1.619 a 785 ha), y los pastos permanentes un 68,7\% (de 916 a 287 ha). Este tipo de medios -sobre todo los pastos permanentes- son los que ocupa la calandria común (Fernández de Mendiola \& Bea, 1998), de modo que la reducción de su superficie ha podido jugar un papel determinante en el declive de la especie.

La cabaña de ovino ha sufrido asimismo un declive del 78\%, pasando de 8.480 cabezas en 1989 a 1.881 en 2009, lo que posiblemente haya favorecido un aumento de la cobertura y volumen de la vegetación de los pastos y matorrales xerófilos. La disminución de la carga ganadera constituye una de las principales amenazas a las que se enfrenta la avifauna esteparia ibérica (Santos \& Suárez, 2005), y en el caso de la Rioja Alavesa podría haber intervenido en el descenso poblacional de algunas especies que ocupan parches de vegetación natural y buscan su alimento en el suelo, caso del alcaudón real 
(Madroño et al., 2004) o el escribano hortelano (Menz et al., 2009; Menz \& Arlettaz, 2012; Sirami et al., 2007).

Nuestros datos hacen referencia a un pequeño grupo de especies y, por tanto, no cabe deducir un descenso generalizado de la avifauna del área de estudio paralelo a los cambios que se han producido en el paisaje. Sin embargo, el predominio de descensos poblacionales observado sí revela un deterioro de la capacidad de acogida del medio para algunas de las especies con mayor interés de conservación. En el suroeste de Europa, la tendencia regresiva de las aves mediterráneas propias de paisajes abiertos parece ser un fenómeno generalizado, que junto con el incremento de aquellas de origen eurosiberiano da lugar a un proceso de homogeneización de las comunidades de aves (Preiss et al., 1997; Seoane \& Carrascal, 2008; Sirami et al., 2007, 2008). El carácter generalista de la mayoría de las especies con poblaciones en aumento refuerza esta tendencia al empobrecimiento y uniformización de la avifauna (Devictor et al., 2008; Kerbiriou et al., 2009; Le Viol et al., 2012). El mantenimiento de la heterogeneidad ambiental y de las prácticas agrícolas tradicionales resultan medidas eficaces para atajar estas tendencias (Benton et al., 2003; Doxa et al., 2012) y, en el área de estudio, actuaciones tales como la recuperación del pastoreo en los parches de vegetación natural y, posiblemente, el mantenimiento de la cobertura herbácea en los viñedos (Sierro \& Arlettaz, 2003; Duarte et al., 2014) podrían tener un efecto beneficioso de cara a incrementar la diversidad y originalidad de su avifauna.

\section{Agradecimientos}

A José María Fernández, que nos hizo llegar diversas observaciones de las especies tratadas en el presente artículo, y que con sus sugerencias mejoró sustancialmente una primera versión del mismo. A David Alday, por su colaboración en la realización del mapa de la figura 1. También a Ramón Arambarri y Fran Silván, que aportaron información sobre diferentes especies. Los comentarios de Emilio Barba, Javier Seoane y un revisor anónimo sirvieron para mejorar numerosos aspectos del manuscrito original.

\section{Bibliografía}

- Álvarez, J., Bea, A., Faus, J.M., Castién, E., Mendiola, I. 1985. Atlas de los vertebrados continentales de Álava, Vizcaya y Guipúzcoa (excepto Chiroptera). Gobierno Vasco. Vitoria-Gasteiz.

- Anton, M., Estrada, J., Herrando, S. 2013. The Red List of Catalan breeding birds (NE Iberian Peninsula) 2012. Rev. Catalana d'Ornitologia 29: 1-19.

- Aseginolaza, C., Gomez, D., Lizaur, X., Montserrat, G., Morante, G., Salaverria, M.R., Uribe-Echebarria, P.M., Alejandre, J.A. 1984. Catálogo florístico de Alava, Vizcaya y Guipúzcoa. Gobierno Vasco. Vitoria-Gasteiz. 
- Aseginolaza, C., Gómez, D., Lizaur, X., Montserrat, G., Morante, G., Salaverria, M., Uribe-Echebarria, P. M. 1988. Vegetación de la Comunidad Autónoma del País Vasco. Gobierno Vasco. Vitoria-Gasteiz.

- Benton, T.G., Vickery, J.A., Wilson, J.D. 2003. Farmland biodiversity: is habitat heterogeneity the key? Trends Ecol. Evol. 18: 182-188.

- Buchanan, G. M., Pearce-Higgins, J.W., Grant, M.C. 2006. Observer variation in estimates of Meadow Pipit Anthus pratensis and Skylark Alauda arvensis abundance on moorland. Bird Study 53: 92-95.

- Devictor, V., Julliard, R., Clavel, J., Jiguet, F., Lee, A., Couvet, D. 2008. Functional biotic homogenization of bird communities in disturbed landscapes. Glob. Ecol. Biogeogr. 17: 252-261.

- Díaz, M., Naveso, M.A., Rebollo, E. 1993. Respuesta de las comunidades nidificantes de aves a la intensificación agrícola en cultivos cerealistas de la Meseta Norte (Valladolid-Palencia, España). Aegypius 11: 1-6.

- Donald, P.F., Green, R.E., Heath, M.F. 2001. Agriculture intensification and the collapse of Europe's farmland bird populations. Proc. Royal Soc. London. Series B 268: 25-29.

- Donald, P. F., Sanderson, F. J., Burfield, I. J, van Bommel, F. P. J. 2006. Further evidence of continent-wide impacts of agricultural intensification on European farmland birds, 1990-2000. Agriculture, Ecosystems \& Environment 116: 189-196.

- Doxa, A., Paracchini, M.L., Pointereau, P., Devictor, V., Jiguet, F. 2012. Preventing biotic homogenization of farmland bird communities: the role of high nature value farmland. Agric., Ecosyst. Environ. 148: 83-88.

- Duarte, J., Farfán, M.A., Fa, J.E., Vargas, J.M. 2014. Soil conservation techniques in vineyards increase passerine diversity and crop use by insectivorous birds. Bird Study 61: 193-203.

- Dubois, P.J., Le Maréchal, P., Olioso, G., Yésou, P. 2008. Nouvel inventaire des Oiseaux de France. Delachaux et Niestlé. París.

- Escandell, V. 2013. Programa Sacre: Tendencia de las aves en primavera. En: Programas de seguimiento de avifauna de SEO/BirdLife en 2012. SEO/BirdLife: 4-9. SEO/BirdLife. Madrid.

- Estrada, J., Pedrocchi, V., Brotons, L., Herrando, S. (Eds.). 2004. Atles dels ocells nidificants de Catalunya 1999-2002. Lynx. Barcelona.

- Fernández, J.M., Gainzarain, J.A. 2004. Deriving avian population trends from atlas data in Spain: opportunities and biases at a regional scale. Bird Census News 17: 5-22.

- Fernández de Mendiola, J.A., Bea, A. (Eds.). 1998. Vertebrados continentales. Situación actual en la Comunidad Autónoma del País Vasco. Gobierno Vasco. Vitoria-Gasteiz.

- Gainzarain, J.A. 1996. Selección de hábitat de la avifauna en una comarca agrícola del Alto Valle del Ebro (Norte de España). Munibe, Cienc. Nat. 48: 3-16.

- Gainzarain, J.A. 2011. Zarcero bereber Hippolais opaca. Noticiario Ornitológico. Ardeola 58: 509. 
- Gainzarain, J.A., Pérez de Ana, J.M. 1995. Distribución y hábitat de la Curruca cabecinegra (Sylvia melanocephala) en las comunidades autónomas del País Vasco y Cantabria. Munibe, Cienc. Nat. 47: 115-118.

- Gámez, I., Serradilla, J., Aguilar, C.M., Robres, F.J., Gutiérrez, Ó. 2010. Anuario Ornitológico de La Rioja. 2004-2008. Zubía Monográfico 21-22: 7-184. Instituto de Estudios Riojanos. Logroño.

- Gobierno Vasco. 2007. Mapa de hábitats del País Vasco. Sistema de clasificación EUNIS. Disponible en Web: http://www.geo.euskadi.net/s69-geodir/es/contenidos/ds_geograficos/habitats/es_opendata/indice.html.

- Greenwood, J.J.D. 1991. Estimating the total number and its confidence limits. Apéndice de: Shrubb, M., Lack, P.C. The numbers and distribution of Lapwings V. vanellus nesting in England and Wales in 1987. Bird Study 38: 20-37.

- Gregory, R.D., van Strien, A., Vorisek, P., Meyling, A.W.G., Noble, D.G., Foppen, R.P.B., Gibbons, D. W. 2005. Developing indicators for European birds. Philosophical Trans. Royal Soc. B 360: 269-288.

• Gutiérrez, R., de Juana, E., Lorenzo, J.A. 2012. Lista de las aves de España. Edición de 2012. Versión online 1.0. SEO/BirdLife.

- Huntley, B., Green, R.E., Collingham, Y., Willis, S.G. 2007. A climatic atlas of European breeding birds. Durham University, RSPB y Lynx Edicions. Durham, Sandy y Barcelona.

- Kampichler C., van Turnhout, C.A.M., Devictor. V., van der Jeugd, H.P. 2012. Large-scale changes in community composition: determining land use and climate change signals. PLoS ONE 7(4): e35272. doi:10.1371/journal.pone.0035272.

- Kerbiriou, C., LeViol, I., Jiguet, F., Devictor, V. 2009. More species, fewer specialists: 100 years of changes in community composition in an island biogeographical study. Divers. Distrib. 15: 641-648.

- Le Viol, I., Jiguet, F., Brotons, L., Herrando, S., Lindström, A., Pearce-Higgins, J.W., Reif, J., Van Turnhout, C., Devictor, V. 2012. More and more generalists: two decades of changes in the European avifauna. Biol. Lett. 8: 780-782.

- Loidi, J., Herrera, M. y Biurrun, I. 1994. Datos sobre la vegetación del País Vasco y zonas limítrofes. (La vegetación del Parque Natural de Valderejo). Gobierno Vasco. Vitoria-Gasteiz.

- Madroño, A., González, C., Atienza, J.C. (Eds.). 2004. Libro Rojo de las Aves de España. Dirección General para la Biodiversidad-SEO/BirdLife. Madrid.

- Martí, R., del Moral, J.C. (Eds.). 2003. Atlas de las aves reproductoras de España. Dirección General de Conservación de la Naturaleza- Sociedad Española de Ornitología. Madrid.

- Martínez, C., Tapia, G.C. 2002. Density of the Little Bustard Tetrax tetrax in relation to agricultural intensification in Central Spain. Ardeola 49: 301-304.

- Menz, M.H.M., Brotons, L., Arlettaz, R. 2009. Habitat selection by Ortolan Buntings Emberiza hortulana in post-fire succession in Catalonia: implications for the conservation of farmland populations. Ibis 151: 752-761. 
- Menz, M.H.M., Arlettaz, R. 2012. The precipitous decline of the ortolan bunting Emberiza hortulana: time to build on scientific evidence to inform conservation management. Oryx 46: 122-129.

- Nuevo, J.A. 1990. Avifauna nidificante en los medios agrícolas de la provincia de Álava. Est. Museo Cienc. Nat. Álava 5: 137-145.

- Preiss, E., Martin, J.L., Debussche, M. 1997. Rural depopulation and recent landscape changes in a Mediterranean region: consequences to the breeding avifauna. Lands. Ecol. 12: 51-61.

- Olsson, U., Alström, P., Svensson, L., Aliabadian, M., Sundberg, P. 2010. The Lanius excubitor (Aves, Passeriformes) conundrum-Taxonomic dilemma when molecular and non-molecular data tell different stories. Mol. Phylogenetics Evol. 55: 347-357.

- R Core Team. 2013. R: A language and environment for statistical computing. R Foundation for Statistical Computing, Viena (Austria).

- Román, J., Román, F., Ansola, L.M., Palma, C., Ventosa, R. 1996. Atlas de las aves nidificantes de la provincia de Burgos. Caja de Ahorros del Círculo Católico. Burgos.

- Sáenz de Buruaga, M., Onrubia, A., Fernández-García, J.M., Campos, M.A., Canales, F., Unamuno, J.M. 2012. Breeding habitat use and conservation status of the turtle dove Streptopelia turtur in northern Spain. Ardeola 59: 291-300.

- Sanderson, F.J., Donald, P.F., Pain, D.J., Burfield, I.J., van Bommel, F.P.J. 2006. Long-term population declines in Afro-Palearctic migrant birds. Biol. Conserv. 131: 93-105.

- Santos, T., Suárez, F. 2005. Biogeography and population trends of Iberian steppe birds. En: Ecology and conservation of steppe-land birds. G. Bota., M.B. Morales, S. Mañosa, J. Camprodon (Eds.): 69-102. Lynx Edicions \& Centre Tecnològic Forestal de Catalunya. Barcelona.

- Seoane, J., Carrascal, L.M. 2008. Interspecific differences in population trends of Spanish birds are related to habitat and climatic preferences. Glob. Ecol. Biogeogr. 17: 111-121.

- Sierro, A., Arlettaz, R. 2003. L'avifaune du vignoble en Valais central: évaluation de la diversité à l'aide de transects. Nos Oiseaux 50: 89-100.

- Sirami, C., Brotons, L., Martin, J.L. 2007. Vegetation and songbird response to land abandonment: from landscape to census plot. Divers. Distrib. 13:42-52.

- Sirami, C., Brotons, L., Burfield, I., Fonderflick, J., Martin, J.L. 2008. Is land abandonment having an impact on biodiversity? A meta-analytical approach to bird distribution changes in the north-western Mediterranean. Biol. Conserv. 141: 450-459.

- Suárez, F. 2004. Aves y agricultura en España peninsular: una revisión sobre el estado actual de conocimiento y una previsión sobre su futuro. En: La ornitología hoy. Homenaje al profesor Francisco Bernis Madrazo. J.L. Tellería (Ed.): 223-265. Editorial Complutense. Madrid.

- Suárez, F., Garza, V., Morales, M.B. 2002. Habitat use of two sibling species, the Short-toed Calandrella brachydactyla and the lesser Short-toed C. rufescens Larks, in mainland Spain. Ardeola 49: 259-272.

-Swihart, R.K., Lusk, J.J., Duchamp, J.E., Rizkalla, C.E., Moore, J.E. 2006. The roles of landscape context, niche breadth, and range boundaries in predicting species responses to habitat alteration. Divers. Distrib. 12: 277-287. 
- Tellería, J.L. 1986. Manual para el censo de los vertebrados terrestres. Ed. Raíces. Madrid.

-Wotton, S.R., Langston, R.H.W., Gibbons, D.W., Pierce, A.J. 2000. The status of the Cirl Bunting Emberiza cirlus in the UK and the Channel Islands in 1998. Bird Study 47: 138-146.

\section{वै०}

- Fecha de recepción/Date of reception: 27/10/2014

- Fecha de aceptación/Date of acceptance: 06/03/2015

Editor Asociado/Associate editor: Emilio Barba 
Apéndice 1.- Mapas de distribución en La Rioja Alavesa de las especies consideradas en el presente trabajo. Se indica mediante puntos su presencia en las cuadrículas UTM de $100 \mathrm{~km}^{2}$ según los datos obtenidos en las primaveras de 2012 y 2013.

Appendix 1.- Maps showing the distribution in Rioja Alavesa of the bird species included in this article, derived from the data obtained in 2012-13 springs. Points indicate their presence at the scale of $100 \mathrm{~km}^{2}$ UTM squares.

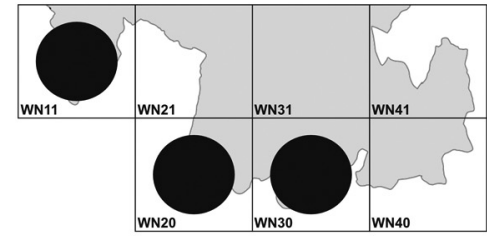

C. oenas

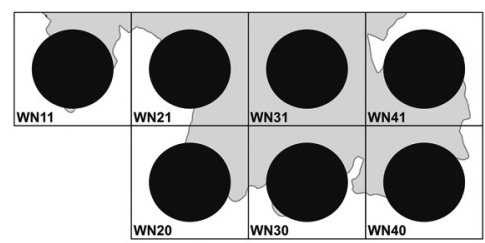

M. apiaster

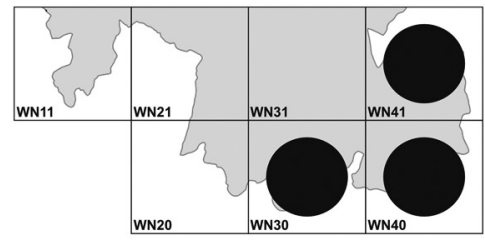

M. calandra

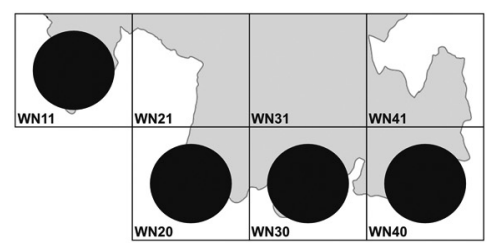

G. theklae

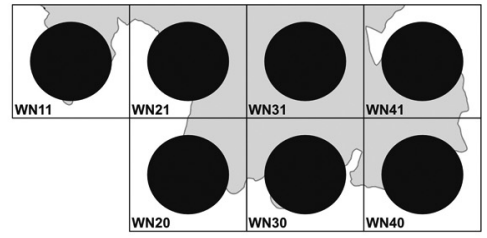

S. turtur

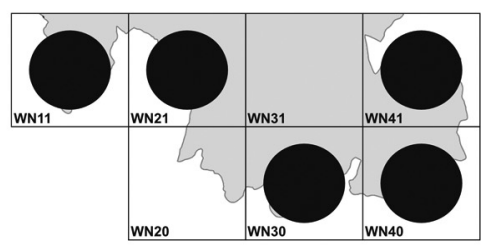

U. epops

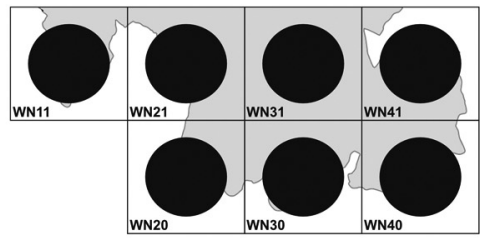

C. brachydactyla

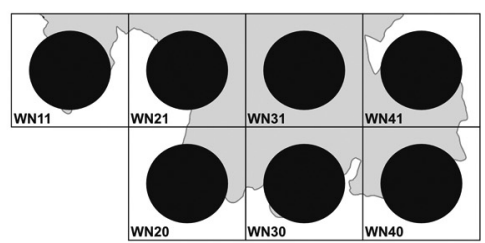

G. cristata 


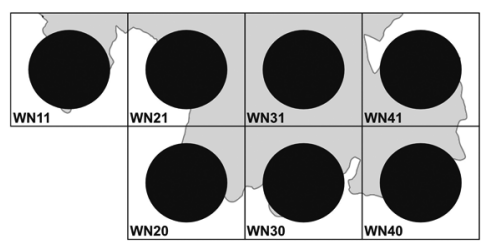

A. campestris

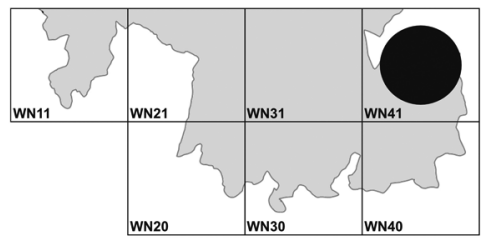

I. opaca

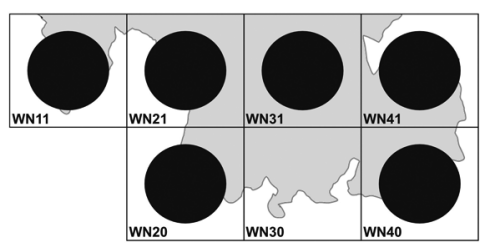

S. cantillans

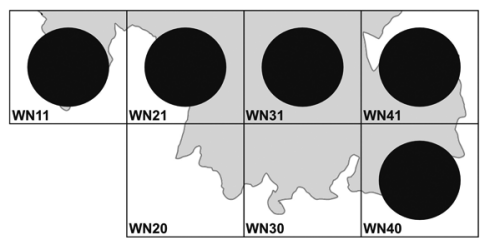

S. hortensis

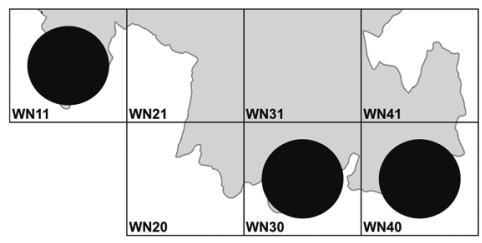

L. senator

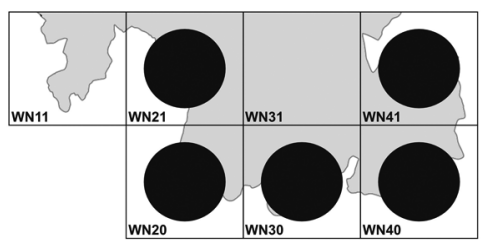

O. hispanica

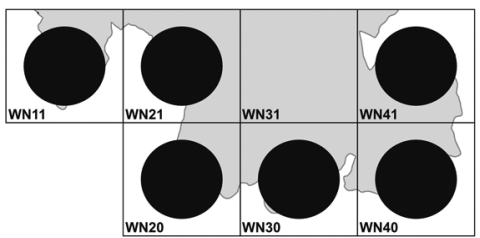

S. undata

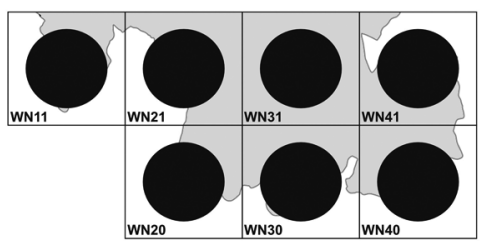

S. melanocephala

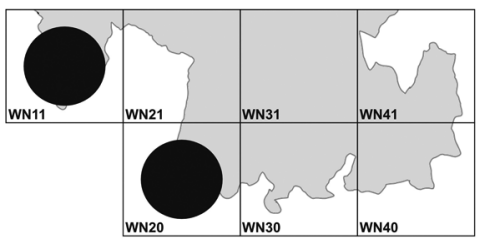

R. pendulinus

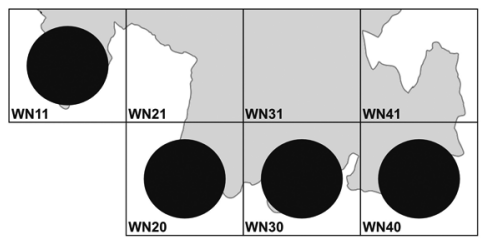

E. hortulana 